INPLASY PROTOCOL

To cite: Yue et al. Effects and safety of perioperative intravenous magnesium on spine surgeries: a protocol of a systematic review and metaanalysis of randomized controlled trials. Inplasy protocol 202170023. doi: 10.37766/inplasy2021.7.0023

Received: 09 July 2021

Published: 09 July 2021

Corresponding author: Lei Yue

yueleimail@foxmail.com

Author Affiliation:

Peking University First Hospital.

Support: Beijing Municipal Natural Science.

Review Stage at time of this submission: Preliminary searches.

Conflicts of interest: None declared.

\section{Effects and safety of perioperative intravenous magnesium on spine surgeries: a protocol of a systematic review and meta-analysis of randomized controlled trials}

Yue, L1; Lin, Z2; Mu, G3; Sun, $\mathrm{H}^{4}$.

Review question / Objective: (1) Systematic uses of magnesium on general anesthesia has shown advantages in terms of reducing intra-operative stress, anesthetics and side-effects and post-operative analgesic use (2)Compared with other studies, spine surgeries typically involve higher technical challenges, more bleeding and more severe postoperative pain and analgesic consumptions. (3) Several randomized controlled trials in recent year have replicated these advantages in the use of intravenous magnesium in spinal surgeries. (4) There is currently a lack of literature synthesis in the safety and effects of systematic use of magnesium on spinal surgeries.

Condition being studied: Spine surgeries, including discectomy, laminectomy, spinal fusion for various spinal diseases.

INPLASY registration number: This protocol was registered with the International Platform of Registered Systematic Review and Meta-Analysis Protocols (INPLASY) on 09 July 2021 and was last updated on 09 July 2021 (registration number INPLASY202170023).

\section{INTRODUCTION}

Review question / Objective: (1) Systematic uses of magnesium on general anesthesia has shown advantages in terms of reducing intra-operative stress, anesthetics and side-effects and post-operative analgesic use (2)Compared with other studies, spine surgeries typically involve higher technical challenges, more bleeding and more severe post-operative pain and analgesic consumptions. (3) Several randomized controlled trials in recent year have replicated these advantages in the use of 
intravenous magnesium in spinal surgeries. (4) There is currently a lack of literature synthesis in the safety and effects of systematic use of magnesium on spinal surgeries.

Condition being studied: Spine surgeries, including discectomy, laminectomy, spinal fusion for various spinal diseases.

\section{METHODS}

Participant or population: Studies will be selected for inclusion if their participants meet specific criteria: (1)Patients who underwent spine surgeries, including discectomy, laminectomy, spinal fusion for various spinal diseases. (2)General anesthesia was chosen for the surgery and tracheal intubation was implemented. (3)Mean times of operation duration shorter than $\mathbf{3 0 0} \mathrm{min}$. We will exclude studies that included participants with: Severe accompanying complications (ASA $>3$ ) Involved other anesthesia methods i.e., regional anesthesia or spinal anesthesia Participants who underwent non-spinal surgeries.

Intervention: Studies evaluating the effect of systematic use of magnesium, only intravenously, as independently or in combination will be considered as target interventions. Intramuscular or intraspinal used of magnesium will not be considered as target interventions.

Comparator: This systematic review will only include studies with comparisons of clear contrast for the index intervention, so that the independent effects of the intervention can be assessed. Studies whose control group received placebo treatment (normal saline) or other comparative treatments will be considered as appropriate comparators.

Study designs to be included: Only randomized controlled trials.

Eligibility criteria: Studies will be selected for inclusion if their participants meet specific criteria: (1)Patients who underwent spine surgeries, including discectomy, laminectomy, spinal fusion for various spinal diseases. (2)General anesthesia was chosen for the surgery and tracheal intubation was implemented. (3)Mean times of operation duration shorter than $300 \mathrm{~min}$. We will exclude studies that included participants with: Severe accompanying complications (ASA $>3$ ) Involved other anesthesia methods i.e., regional anesthesia or spinal anesthesia Participants who underwent non-spinal surgeries.

Information sources: English Databases: PubMed, Embase, Web of science, SCOPUS. Trial registries: ClinicalTrials.gov. Grey literature: Google scholar. The reference lists of retrieved trials and previous systematic reviews will be searched for citation of potentially eligible trials. In case that any questions about trials arise, the corresponding author of articles will be contacted.

Main outcome(s): Pain intensity at $24 \mathrm{~h}$ : VAS or NRS scales. Analgesics dosage in the first $24 \mathrm{~h}$ : $\mathrm{mg}$ of parenteral morphine.

Additional outcome(s): Cumulative dose of anesthetics of the operation. Blood loss of the surgery. Time of tracheal extubation. Time of recovery (orientation and response to verbal commands). Incidence of nonneurologic adverse events (hypotension, bradycardia, postoperative nausea and vomiting (PONV).

Quality assessment / Risk of bias analysis: The risk of bias for each included RCTs will be assessed by two reviewers (Y.L. and M.G.Z.) independently using the bias tool recommended by the Cochrane Back and Neck (CBN) Group, and the overall quality of each included trials will be assessed by Jaded score. Any disagreement will be resolved by consensus of the whole group. The graphical presentation of assessment of risk of bias will be generated by RevMan 5.3. We will also apply Grading of Recommendations, Assessment, Development, and Evaluation (GRADE) approach to evaluate the overall quality of the evidence based on five domains: limitations of design, inconsistency of 
results, indirectness, imprecision, and other factors (e.g., publication bias). GRADE approach evaluates the quality of evidence as 'high', 'moderate', 'low', or 'very low' by the outcome.

Strategy of data synthesis: (1) The results from finally screened studies will be combined to estimate as effective results in standardized mean differences (SMD) and $95 \% \mathrm{Cl}$ for continuous outcomes. As to dichotomous outcomes, pooled risk ratio (RR) and $95 \% \mathrm{Cl}$ will be estimated. Radom effect model will be used. (2) The synthesis will be done by generating a forest plot of the study estimates. We will evaluate the heterogeneity of the included studies with I $^{2}$ test. Heterogeneity will be examined by $\mathbf{I}^{2}$ value as low, moderate or high $\left(I^{2}\right.$ value of $25 \%, 50 \%$ and $75 \%$ respectively). (3) Statistical significance will be set at $P<0.05$ in this review.

Subgroup analysis: To detect possible heterogeneity of the results, if the sample size is sufficient ( $>400$ participants), subgroup analysis will be performed on the primary outcomes. The subgroup analysis will be performed on the dosage of administered magnesium, which will be determined by post-hoc analysis.

Sensitivity analysis: To assess the robustness of the summary estimates and detect whether any single study represents a large proportion of heterogeneity, sensitivity analyses will be performed by eliminating each included study one by one from the pooled analyses.

Language: English.

Country(ies) involved: China.

Keywords: magnesium, spine surgery, perioperative.

Contributions of each author:

Author 1 - Lei Yue.

Email: yueleimail@foxmail.com

Author 2 - Zengmao Lin.

Email: linzengmao@163.com

Author 3 - Guanzhang Mu.

Email: 2690876523@qq.com
Author 4 - Haolin Sun.

Email: sunhaolin@vip.163.com 